Mathematical Modelling and Analysis

Volume 20 Number 6, November 2015, 701-714

http://dx.doi.org/10.3846/13926292.2015.1108250

(c) Vilnius Gediminas Technical University, 2015
Publisher: Taylor\&Francis and VGTU

http://www.tandfonline.com/TMMA

ISSN: $1392-6292$

eISSN: $1648-3510$

\title{
On Efficient Numerical Algorithms for Simulation of High Power Electrical Cables
}

\section{Raimondas Čiegis, Gerda Jankevičiūtè and Natalija Tumanova}

\author{
Vilnius Gediminas Technical University \\ Saulètekio al. 11, LT-10223 Vilnius, Lithuania \\ E-mail(corresp.): rc@vgtu.It \\ E-mail: gerda.jankeviciute@vgtu.It \\ E-mail: natalija.tumanova@vgtu.lt
}

Received September 22, 2015; revised October 6, 2015; published online November 15, 2015

\begin{abstract}
The new virtual modelling tool is constructed, which is used for optimal design of power transmission lines and cables. The construction of such lines should meet the latest power transmission network technical and economical requirements. The solver is is based on classical and modified mathematical models describing main heat conduction processes: diffusion, convection and radiation in various materials and environments. In basic heat conduction equation, we take into account a linear dependence of the resistance on temperature. Multi-physic and multi-scale models are required to simulate industrial cases of power transmission lines. The velocity of convective transport of the heat in air regions is simulated by solving a coupled thermo-convection problem including the heat conduction problem and the standard Navier-Stokes model of the heat flow in air. Another multi-physic model is used to describe changes of material heat conduction coefficients in soil due to influence of heating. This process is described by by solving a simplified mass balance equation for flows in porous media. The multi-scale and homogenization analysis is required to to formulate simple and accurate mathematical describing heat conduction process is metal region which consists of a bundle of tightly coupled metal wires. The FVM is used to solve the obtained systems of differential equations. Discretization of the domain is done by applying "aCute" mesh generator, which is a modification of the well-known Triangle mesh generator. The discrete schemes are implemented by using the OpenFOAM tool.
\end{abstract}

Keywords: heat conduction, multi-scale model, multi-physics, homogenized model, finite volume method.

AMS Subject Classification: 65M08; 65Z05; 80A20.

\section{Introduction}

This research is aimed to develop design rules for power transmission lines and cables, which have to meet the latest power transmission network technical 
and economical requirements. At present the power lines are over-dimensioned by up to $60 \%$ in terms of transmitted power. However, today, as the new distributed generating capacities are installed e.g. large wind farms, bio-gas plants or waist-to-energy plants, the infrastructure of power grid must be redesigned or new optimization strategies for the available grid developed. Power cables for power distribution applications are still rated according to IEC 287 and IEC 853 standards, which use the Neher and McGrath methods from $1957[1,15]$. However, today there are many applications, where analytical and heuristic formulas cannot describe precisely enough the conditions under which the cables are installed. An example could be an underground cable route, where the installation conditions for a cable are different only for a short distance including only a short crossing of the road. The present standards require that the cable's current-carrying capacity must be reduced due the worst case conditions. Today the cost effective designing of cable installations comes us an urgent need, since the copper price level has reached its maximum since decades. At the same time the safety of the design is also must be guaranteed. Thus a direct simulation of cables including all specific conditions under which the cables are operating is needed. The knowledge of dynamics (in time) of heat distribution in/around electrical cables is necessary to optimize the usage of electricity transferring infrastructure. It is important to determine various parameters of the cable networks: maximal electric current for the cable; optimal cable parameters in certain circumstances; cable life expectancy and many other engineering factors.

The main aim of this paper is to construct accurate and robust numerical solvers for simulation of heat transfer in eletrical cables. Some preliminary results of this paper are presented in our short paper [4].

The rest of this paper is organized as follows. In Section 2, the mathematical models of electrical cables in various environments are presented. They describe various layouts of cables directly buried in soil, cables in pipes and the pipes directly buried in soil, cables in air. Heat transfer models are defined and the heat source mainly depends on the resistivity of the electrical current and is described by the Joule-Lenz law. Additional source of the heat is described by heating effect of the sun.

In Section 3, numerical algorithms are described. The solvers are constructed by using the Finite Volume Method (FVM). Discretization of the domain is done by applying "aCute" mesh generator, which is a modification of the well-known Triangle mesh generator. The discrete schemes are implemented by using the OpenFOAM tool.

Some results of numerical experiments are presented in Section 4. One robust and efficient numerical algorithm is presented to find the maximal value of electrical current for a specified maximal temperature of cables. Such information is most important for engineers in construction of networks of electrical cables.

Multi-scale structures should be simulated in metal region of cables. In Section 5 simplified averaged mathematical models are derived, homogenization of the conductance region of electrical cables is done by defining heuristically new homogeneous metal domains with some virtual materials filling these do- 
mains. Results of computational experiments are presented and the accuracy of homogenized models is investigated.

\section{Mathematical Models}

In this section we formulate main mathematical models of heat transfer in underground electrical cables. We will restrict only to those cases, which are most important for typical layouts of industrial cable networks. A good review on such mathematical models is presented in $[9,13]$. Thus we will present only a short description of basic details on high voltage cables and their installation conditions. Figure 1 illustrates a typical high voltage cable, it consists of conductor (most often copper or aluminum), conductor screen, insulation layer (cross-linked polyethylene), insulation screen, metallic shielding (copper tape/wire, aluminum/lead sheath) and outer covering (polyvinyl chloride, polyethylene, nylon) layers.

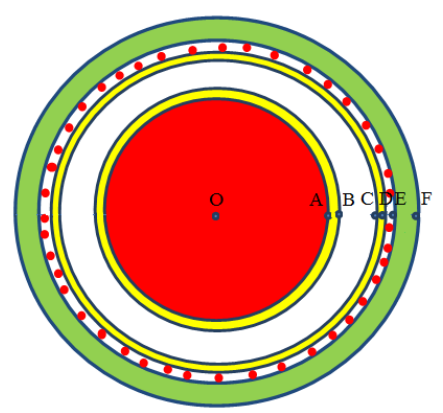

Figure 1. A standard structure of high-voltage cables consisting of six layers.

Different installation environments are considered in real world applications. Here we restrict to simulation of the following environments and layouts of cables:

a) cables in air,

b) cables directly buried in soil,

c) cables are put into pipe and the pipe is directly buried in soil,

d) cable groups (duct banks).

In each case a group of cables can be arranged horizontally or vertically (see, Figure 2).

Next we present basic mathematical models which are used in our simulations of heat transfer in different environments.

\subsection{Heat Transfer in Solids}

It is well-known that electric current flowing through cable generates heat. This process is described by the Joule-Lenz law. The non-stationary heat-transfer is 


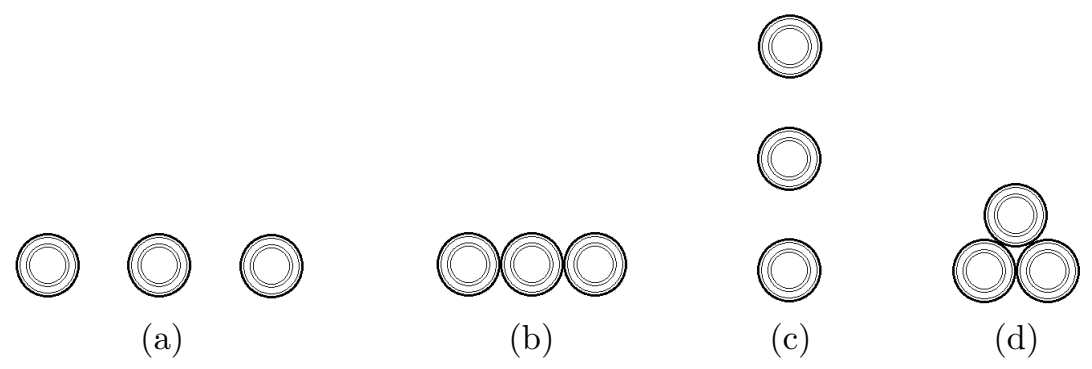

Figure 2. Examples of cables layouts: (a,b) three single core cables are arranged horizontally; (c) three single core cables are arranged vertically; (d) three-core cable.

described by classical heat equations in heterogeneous medium $[2,3]$. Since the bundle of cables consisting of metal cores and various insulation layers is placed in a medium, we assume that the coefficients of thermal conductivity, material density and specific heat capacity are discontinuous piece-constant functions depending on spatial coordinates.

Since the heat transfer mechanism in the underground electrical cables is quite complicated, and many processes can be described only approximately, the structure of cables also can be simplified. In taking any simplification assumptions we should follow one important rule of engineering design - the obtained approximate models should give estimates from above of real experimental values of temperatures. In such a case we can guarantee that the recommendations done on the basis of simulations are safe.

It is typical in applications, that the length of cable is much bigger than its diameter, thus effects along the cables' length can be neglected. A sand or soil area around cables is much larger than cables' area, so usually two-dimensional nonstationary models are sufficient for the analysis.

We note that properties of different filler (materials) should be taken into account. In simple approximations we consider apriori defined regions of sand, wet and dry soil and other materials. In more challenging situations the multiphysic models are used and the flow of water in porous media is simulated in a coupled system of equations.

For heat transfer in underground cables we assume the diffusion to be the main transfer mechanism. A mathematical model of the heat source is described by the Joule-Lenz law. Then the mathematical model of non-stationary heat-transfer is given by the parabolic differential equation $[2,3]$ :

$$
\begin{cases}c \rho \frac{\partial T}{\partial t}=\nabla \cdot(\lambda \nabla T)+q_{0}\left(1+\alpha\left(T-T^{*}\right)\right) I^{2}, & t \in\left[0, t_{\max }\right], x \in \Omega, \\ T(x, 0)=T_{0}, & \text { when } x \in \Omega, \\ T \text { and } \lambda \nabla T \text { are continuous, } & \text { when } x \in \Omega,\end{cases}
$$

here $x=\left(x_{1}, x_{2}\right), \Omega$ is the domain, $T(x, t)$ is temperature in the Kelvin scale, $\lambda(x)>0$ is the heat conductivity coefficient, $q_{0}(x, t, T)$ defines the the source function. Coefficient $\rho(x)>0$ is the mass density, $c(x)>0$ is the specific heat capacity, $\alpha(x)$ is temperature resistance coefficient, $T_{0}$ is the initial tem- 
perature. In the model, we take into account dependence of the resistance on temperature, $T^{*}$ is the reference temperature and $I$ is the electrical current. Due to different properties of materials included into the model, coefficients $\lambda, c, \rho$ are discontinuous at boundaries of different regions, the geometry of these regions can be very complicated, and the values of coefficients may vary thousands times. That makes the simulation task very challenging.

Here we should take into account the fact, that metal regions of the cable are not homogeneous but are a bundle of small wires tightly put together. We still assume that the diffusion of heat is the main transfer mechanism, but a proper homogenization technique should be applied to derive virtual metal regions. Homogenized models will be investigated in the next section.

Various boundary conditions are applied to describe the heat flow through boundaries of the domain $\Omega$, which is rectangular in simulations given below:

- $T(x, t)=T_{b 1}$ for the upper boundary, $T(x, t)=T_{b 2}$ for the lower boundary, $\frac{\partial T}{\partial x_{1}}=0$ for the left and right boundaries are applied to simulate boundary conditions in winter $\left(T_{b 1}=T_{b 2}\right)$ or summer $\left(T_{b 1}>T_{b 2}\right)$.

- Boundary conditions of the third type

$$
\lambda \frac{\partial T}{\partial x_{2}}=\beta(T)\left(T(x, t)-T_{a i r}\right)+\psi(x, t)
$$

are applied on the upper boundary to evaluate the cooling effects of wind in air and heating effect of the sun. The nonlinear coefficient $\beta(T)$ defines the advection and radiation processes.

\subsection{Cable in Pipe}

Figure 3 shows a single cable placed at the bottom of a plastic pipe directly buried in the soil. The plastic pipe is placed in the center of the soil domain. Due to its relatively low heat conduction coefficient a plastic pipe represents

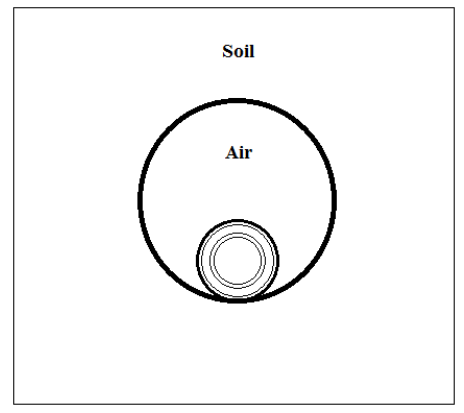

Figure 3. A single cable in directly buried pipe.

a significant thermal resistance for the cooling of the cable. The main heat transfer mechanism in air is described by air circulation inside the plastic pipe. 
Velocities of the free convection process are computed by solving the NavierStokes equations (the conservation of continuity, momentum and energy equations) in the air area. In order to get a simpler mathematical model, we assume the air to be incompressible for small velocities. Then, assuming the velocities are not large, the model of laminar incompressible flow is given by the following system of equations:

$$
\begin{aligned}
& \nabla \cdot \mathbf{u}=0, \\
& \rho \frac{\partial \mathbf{u}}{\partial t}+\rho \mathbf{u} \nabla \cdot \mathbf{u}-\nabla \cdot(\eta \nabla \mathbf{u})=-\nabla p-\rho \tilde{\alpha} \mathbf{g}\left(T-T_{0}\right), \\
& \rho c\left(\frac{\partial T}{\partial t}+\nabla \cdot(\mathbf{u} T)\right)-\nabla \cdot(\lambda \nabla T)=q,
\end{aligned}
$$

where $\mathbf{u}(x, t)$ is velocity of the flow, $p$ is the pressure, $\eta$ is the dynamic viscosity, $\tilde{\alpha}$ is the thermal expansion coefficient.

Then the heat conduction equation (2.4) is extended to the whole domain. In the soil, pipe and cable regions the advection term $\nabla \cdot(\mathbf{u} T)$ is equal to zero, and equation (2.4) is reduced to the basic non-stationary heat conduction equation (2.1).

\subsection{Heat Transfer in Soil}

In most papers dedicated to simulation of heat conduction in electrical cables the soil is considered as solid material. In this subsection we formulate a multi-physic mathematical model of coupled heat and water transfer in variable saturated soil. The porous medium is considered to be rigid and unsaturated, hence two phases are present: liquid (water) and gas (water vapor and air). The temperature of the solid, liquid and gas phases are considered to be in local thermal equilibrium. The Richards equation is obtained from the mass conservation equation for liquid phase and Darcy law $[4,7]$ :

$$
\varepsilon \frac{\partial \rho_{w} S^{l}}{\partial t}+\nabla \cdot\left(-\rho_{w} \vec{K} \frac{k_{r e l}^{l}\left(S^{l}\right)}{\mu_{w}}\left(-\nabla p^{c}\left(S^{l}\right)-\rho_{w} \vec{g}\right)\right)=0,
$$

where $\varepsilon$ is porosity of the porous medium, $\rho_{w}$ is density of the water, $S^{l}$ is saturation of the liquid phase, $p^{c}$ is capillary pressure, $\vec{K}$ is intrinsic permeability tensor of the porous medium, $\mu_{w}$ is viscosity of the water, $\vec{g}$ is vector of gravitational acceleration. In general, the capillary pressure also depends on the temperature $p^{c}=p^{c}\left(S^{l}, T\right)$.

For the description of the heat transfer, the energy conservation is used

$$
(\rho c)_{e f f} \frac{\partial T}{\partial t}+\left(\rho_{w} \vec{K} \frac{k_{r e l}^{l}\left(S^{l}\right)}{\mu_{w}}\left(\nabla p^{c}\left(S^{l}\right)+\rho_{w} \vec{g}\right)\right) \cdot \nabla T=\nabla \cdot\left(\lambda_{e f f} \nabla T\right)+q,
$$

where $(\rho c)_{e f f}$ is the effective heat capacity, $\lambda_{e f f}$ is the effective heat conductivity.

The presented mathematical model is used as a basic template for construction of modified models. Here we shortly note on two possible generalizations. The soil-atmosphere interface is an important boundary condition affecting 
subsurface movement of liquid water and heat under field conditions. Another approach is to use a generalization of the Darcy law, when the flow velocity directly depends also on the gradient of temperature. It should be remarked that the last generalization is considered as an heuristic, which gives the additional degree of freedom to fit the mathematical model to experimental data.

\section{Numerical Approximations}

The obtained systems of PDEs are solved by using Finite Volume Method. OpenFOAM (Open source Field Operation And Manipulation) tool is used to implement the given finite volume schemes. OpenFOAM is a $\mathrm{C}++$ toolbox (library) targeted for the development of customized numerical solvers for partial differential equations [17].

Here we describe briefly two main challenges we have solved in order to adapt this universal and robust tool to simulate heat transfer processes in high power electrical cables.

The first one deals with adaptation of standard library solvers to solve the coupled multi-physics mathematical model (2.2) - (2.4). It consists of different models in different sub-regions and application of OpenFOAM functionality requires some non-trivial modifications of basic models presented in the OpenFOAM library. For mathematical models describing coupled multiphysics problems, two different approaches can be used to design robust and efficient solvers [14]. Monolithic solvers operate directly on the system of nonlinear algebraic equations, obtained after the discretization of the system of PDEs. In the partitioning approach the discrete system is solved by using the single-physics solvers in decoupled fixed-point iterations. A review for a comparison of fixed-point methods is given in [10]. The latter approach is implemented in OpenFOAM. The important original part of our algorithm consists in implementation of FVM scheme for Navier-Stokes equations as an independent standard OpenFOAM solver on an additional mesh, which is embedded into the main discrete mesh. Such approach enables users to solve decoupled equations of the full multi-physics system only on parts of the whole domain, thus reducing computational costs essentially.

The second challenge is connected to selection of the optimal mesh to discretize the given domain and sub-domain describing different types of materials. In order to approximate heat conduction equation in solid medium (2.1), two sub-tasks should be solved accurately.

First, the numerical fluxes of the discrete solution must be orthogonal to the boundary of finite volumes [6]. Quadrilater meshes can be generated by using OpenFOAM native mesh tools, they lead to regular classical five-point stencils in approximation of diffusion operators. The main drawback of such meshes is that orthogonality of fluxes is not preserved automatically and should be obtained by applying additional interpolation corrections. Such modified schemes require iterative implementation even for linear problems. The stability of finite volume schemes also can be violated due to corrections of flux approximations and should be investigated for each case of problems. In our solver this problem is solved by using a proper Delaunay triangulation of the domain. Discretiza- 
tion of the domain is done by applying "aCute" mesh generation tool, which is a modification of the well-known Triangle mesh generator [19]

Second, a special interpolation should be used for definition of discontinuous coefficients $\lambda$ in the discrete diffusion flux term, namely harmonic version of the interpolation formula [6].

\section{Computational Experiments}

In this section we present some results of simulations of heat transfer in electrical cables. Our aim is to demonstrate possibilities of the constructed simulation tool. First we consider the case of one cable which is placed at the bottom of a plastic pipe buried in the soil. We have simulated the heat transfer during three summer months, when the soil is assumed to be semi-dry, boundary conditions are taken $T=293 \mathrm{~K}$ on all boundaries, and the electrical current is equal to $470 \mathrm{~A}$. At the final time the solution practically reached a stationary phase.

Figure 4 shows a distribution of temperature and a velocity field in the domain of computations.

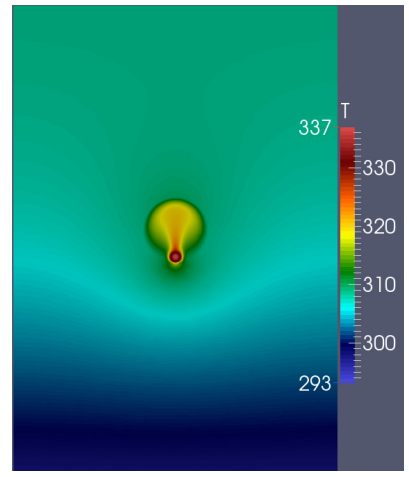

(a)

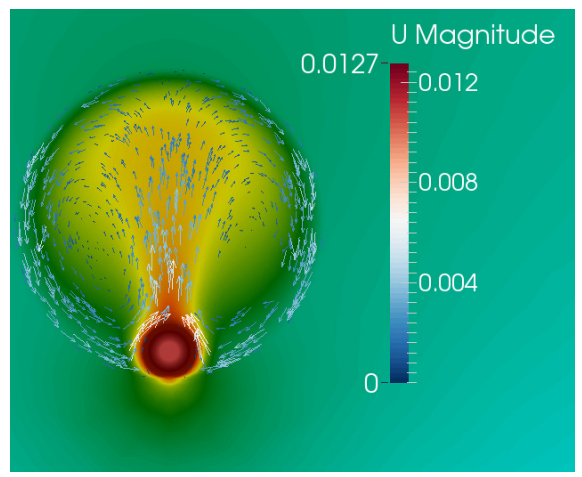

(b)

Figure 4. Simulation results for a single cable in a pipe: (a) distribution of temperature, (b) distribution of velocity fields.

The presented results show that the main mechanism of heat transfer in air is describe by the advection processes, thus accurate simulation of velocity fields is required.

Next we have investigated the influence of different arrangements of cables. Thus we have considered trefoil cables buried directly in soil and preserved all remaining conditions the same as in the previous experiment. Results of computations are given in Figure 5.

The presented results show a strong heating effect on neighboring systems of trefoil cables and therefore the maximal electrical current should be reduced in the case of two pairs of trefoil cables. 


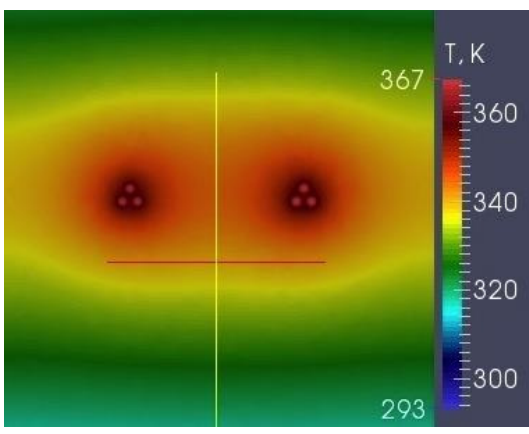

(a)

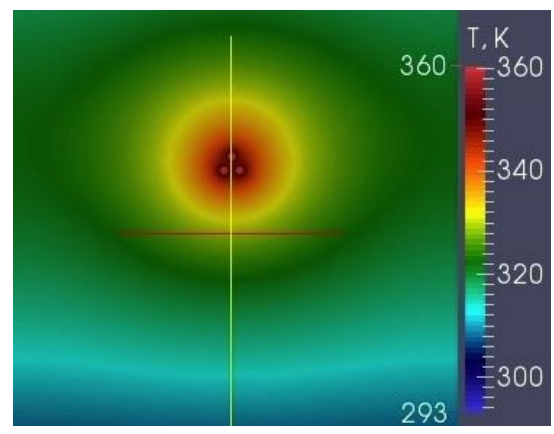

(b)

Figure 5. Simulation results for trefoil cables: (a) electrical current $I=609$ A, (b) $I=675$ A.

Determination of the maximal electrical current value. In engineering practice, it is expected to get heat transfer simulation results in the form

$$
I_{\max }=F\left(T_{\max }\right),
$$

where $T_{\max }$ is the maximal allowed temperature of cables and $I$ is the maximal possible value of electrical current such that

$$
T(x, t) \leq T_{\max }, \quad x \in \Omega .
$$

Such a form of the solution can be achieved if we get the analytical solution of simplified mathematical models. In order to provide a similar information for general more accurate mathematical models we apply the numerical approach.

Since engineers are interested in the upper bound of maximal electrical current values, then using the well known maximum principle for parabolic equations we consider the stationary case of the mathematical model for heat conduction in electrical cables

$$
\begin{aligned}
& -\nabla \cdot(\lambda \nabla T)=q_{0} I^{2}\left(1+\alpha\left(T-T_{b}\right)\right) \quad x \in \Omega, \\
& \left.T(x)\right|_{\partial \Omega}=T_{b} .
\end{aligned}
$$

The source term depends on the solution $T$. Taking into account this linear dependence, it is convenient to rewrite mathematical model (4.1) as elliptic problem

$$
\begin{aligned}
& L T:=-\nabla \cdot(\lambda \nabla T)+q(x) T=f(x), \quad x \in \Omega, \\
& \left.T(x)\right|_{\partial \Omega}=T_{b},
\end{aligned}
$$

the source function and sink term $q(x)$ are defined as

$$
f(x)=\left\{\begin{array}{l}
q_{0} I^{2}\left(1-\alpha T_{b}\right), \quad x \in \Omega_{M}, \quad q(x)=\left\{\begin{array}{l}
-q_{0} \alpha I^{2}, \quad x \in \Omega_{M}, \\
0, \quad x \notin \Omega_{M},
\end{array} \quad x \notin \Omega_{M},\right.
\end{array}\right.
$$

where $\Omega_{M}$ indicates the domain of metallic core. 
Next we apply the techniques described above and approximate the differential equation by the standard FVM scheme

$$
\begin{aligned}
& L_{h} T_{h}:=-\nabla_{h} \cdot\left(k \nabla_{h} T_{h}\right)+q(X) T_{h}=f_{h}(X), \quad X \in \Omega_{h}, \\
& \left.T_{h}(X)\right|_{\partial \Omega_{h}}=T_{b} .
\end{aligned}
$$

Since sink/source term $q(x) \leq 0$, thus the existence and convergence of the discrete solution requires a special analysis. The conclusion depends on the relation among intensity of the source term coefficient $q$ and the type of boundary conditions. The dependence monitor is defined by the minimal eigenvalue of the full boundary value problem. We note that a detailed analysis for such problems is done in $[5,12]$ and these results can be applied for problem (4.3).

The obtained system of linear equations is solved efficiently by the preconditioned conjugate gradient iterative method and algebraic multigrid method is used as the preconditioner $[16,20]$.

From mathematical model (4.3) by fixing the value of electrical current $I$ we compute the distribution of temperature $T(x)$ and find the maximal value of it

$$
T_{\max }=G(I)
$$

As was stated above, for engineers it is important to get the maximal possible value of electrical current for a specified bound of the temperature in domain $\Omega$. Thus we should solve the nonlinear equation (4.4) and find $I$ for a given $T_{\max }$. By using the maximum principle and comparison theorems it is easy to prove (see $[5])$ that $T_{h}(X, I)$ depends monotonically on $I$, i.e.

$$
\frac{\partial T_{h}(X, I)}{\partial I} \geq 0, \quad X \in \Omega_{h}
$$

Thus equation (4.4) can be solved efficiently by the classical bisection method. In Table 1 we present results of computational experiments. We have simulated the heat transfer in a one electrical cable which was buried directly in the dry soil. The critical values of the electrical current $I$ in $A$ are computed for different threshold values of temperature $T_{\max }$. The discrete mesh consisted of 25100 triangles. The computations were done on the Vilkas cluster at Vilnius Gediminas technical university. It consists of eight Intel Quad i7-860 processors $(2.80 \mathrm{GHz})$ interconnected via Gigabit Smart Switch (http://vilkas.vgtu.lt). The CPU time of finding electrical current value $I$ for a given $T_{\max }$ is approx. 1.3 seconds.

Table 1. Analysis of maximal values of electrical current: $I$ denotes the largest possible value of electrical current, $T$ denotes the threshold value of temperature.

\begin{tabular}{rrrrr}
\hline & $T=70$ & $T=80$ & $T=90$ & $T=100$ \\
\hline$I$ & 1047.5 & 1128.5 & 1200.7 & 1264.1 \\
\hline
\end{tabular}




\section{Homogenized heat conduction model}

In this section we investigate the accuracy of simplified mathematical models of high power electrical cables obtained after application of homogenization technique. As was stated above the metal region of electrical cables consists of tightly bundled copper wires and should be considered as composite material. We note, that accurate direct numerical simulation of such complicated structures should be avoided since:

- The size of a discrete problem and CPU time would increase essentially;

- It is not efficient and appropriate to simulate very accurately one specific process if simplifying assumptions (and approximation errors) are introduced at the remaining parts of the model. The optimality principle requires to balance all approximation errors.

A general theory of multi-scale analysis is quite well developed and homogenization of heat conduction models for composite materials can be done by using two-scale analysis technique $[8,11,18]$.

In this section we propose to take into account specific details of composite materials defining electrical cables: the grain scale is not well separated from the macro-scale and heat transfer in air domains is simplified to virtual diffusion process with some small diffusion coefficient. Thus the main heat transfer flow is defined by metal regions. Homogenization of the mathematical model of electrical cables is done by defining heuristically new geometrical domains of metal. We put the following main requirements to the averaging procedure:

- A total amount of produced heat should be preserved as in the original mathematical model;

- Solutions of simplified mathematical models should give an accurate estimates from above of the benchmark model solution.

In order to compare different homogenization strategies we consider the following benchmark domain presented in Figure 6 . In conductor domain $\Omega_{C}=$ $\Omega_{M} \cup \Omega_{A}$ quadratic wires are connected by thin bridges. Let denote the copper wires domain by $\Omega_{M}$. Air regions $\Omega_{A}$ are filled with a virtual material for which the heat diffusion coefficient $\lambda$ is much smaller than in the metal region.

We consider two homogenized models of electrical cables.

HM1. The conductor domain is reduced to metal wires domain. An additional ring is filled with some material, the diffusion coefficient of which can be fitted to experimental data. We consider two cases when this region is filled with standard isolator or with some virtual material. No current is flowing through this ring region.

HM2. The conductor region is filled with metal and the electrical current is flowing through it. In order to conserve the total amount of heat produced by this current we should reduce the intensity of the current $\widetilde{I}$ as:

$$
\frac{\widetilde{I}^{2}}{A_{C}^{2}}=\frac{A_{M}}{A_{C}} \frac{I^{2}}{A_{M}^{2}},
$$




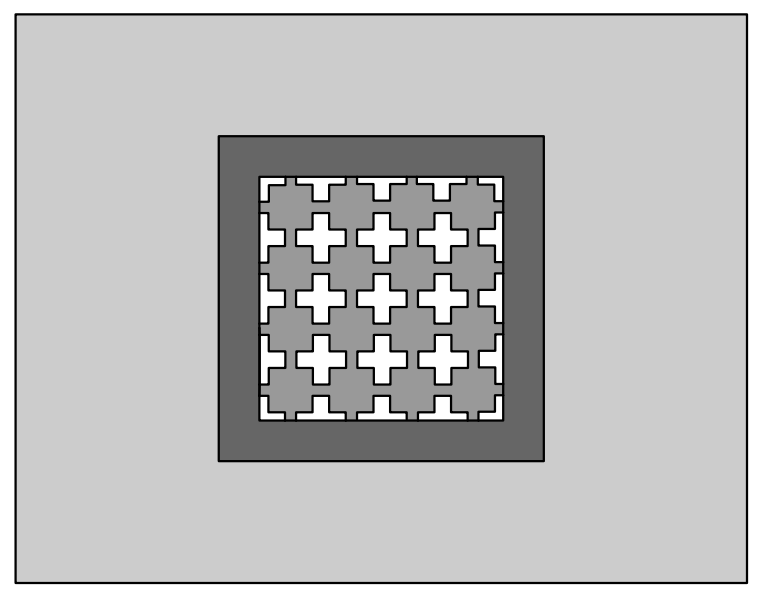

Figure 6. The domain of benchmark problem: conduction domain $\Omega_{C}$ is filled with quadratic wires connected by thin bridges.

where $A_{j}=\left|\Omega_{j}\right|$ denotes the area of domain $\Omega_{j}$.

The benchmark problem is defined by the following geometrical parameters: the width of the conduction domain $\Omega_{C}$ is 0.04 , the width of metal bridges is 0.002 , the metal domain $\Omega_{M}$ fills 0.72 part of the full conduction domain, the width of isolator ring is 0.02 . The diffusion coefficients $\lambda$ of materials (metal, virtual air, isolator, soil, virtual filler) are defined as

$$
\lambda_{M}=387.6, \quad \lambda_{A}=0.15, \quad \lambda_{I}=0.333, \quad \lambda_{S}=1, \quad \lambda_{F}=2 .
$$

Results of computational experiments are given in Table 2. Here we present maximal values of temperature in $\mathrm{K}$ for the benchmark model $M M$ and different homogenized models $H M$. Here $H M 1 a$ denotes the case of model $H M 1$ when the ring is filled with isolator, $H M 1 b$ denotes the case when this ring is filled with with a virtual material. I denotes the value of basic electrical current in A.

Table 2. Comparison of homogenized mathematical models: maximal values of temperature in $\mathrm{K}$ for the benchmark model $M M$ and different homogenized models $H M$. Here $I$ denotes the value of basic electrical current in $\mathrm{A}$.

\begin{tabular}{lcccc}
\hline$I$ & $M M$ & $H M 1 a$ & $H M 1 b$ & $H M 2$ \\
\hline 2400 & 361.9 & 368.5 & 359.7 & 357.6 \\
2600 & 377.8 & 386.4 & 375.0 & 372.3 \\
\hline
\end{tabular}

The presented results show that all heuristics give reasonable accuracy and can be recommended for usage in design of optimal electrical cable networks. Heuristic $H M 1 a$ gives the estimate of the solution from above and thus can be used to define an alternative to requirements defined by standards. 


\section{Acknowledgment}

This work was supported by Eureka project E!6799 POWEROPT "Mathematical modelling and optimization of electrical power cables for an improvement of their design rules".

The authors would like to thank the referee for his constructive criticism, which helped to improve the clarity and quality of this note.

\section{References}

[1] G.J. Anders. Rating of Electric Power Cables in Unfavorable Thermal Environment. IEEE Press Series on Power Engineering. John Wiley \& Sons, New York, 2005.

[2] T.L. Bergman, A.S. Lavine, F.P. Incropera and D.P. DeWitt. Fundamentals of Heat and Mass Transfer. Wiley, 2011.

[3] R. Čiegis, A. Ilgevičius, H. Liess, M. Meilūnas and O. Suboč. Numerical simulation of the heat conduction in electrical cables. Mathematical Modelling and Analysis, 12(4):425-439, 2007.

http://dx.doi.org/10.3846/1392-6292.2007.12.425-439.

[4] R. Čiegis, G. Jankevičiūtè, A. Bugajev and N. Tumanova. Numerical simulation of heat transfer in underground electrical cables. In Progress in Industrial Mathematics at ECMI2014, Mathematics in Industry. Springer, 2015.

[5] R. Čiegis, G. Jankevičiūtè and O. Suboč. Numerical simulation of the heat conduction in composite materials. Mathematical Modelling and Analysis, 15(1):922, 2010. http://dx.doi.org/10.3846/1392-6292.2010.15.9-22.

[6] W. Hundsdorfer and J.G. Verwer. Numerical Solution of Time-Dependent Advection-Difusion-Reaction Equations, volume 33 of Springer Series in Computational Mathematics. Springer Berlin Heidelberg, Berlin, Heidelberg, New York, Tokyo, 2003. http://dx.doi.org/10.1007/978-3-662-09017-6.

[7] T. Izumi. Inverse modeling of variably saturated subsurface water flow in isothermal/non-isothermal soil. Mem. Fac. Agr., Ehime Univ., 2012.

[8] V.V. Jikov, S.M. Kozlov and O.A. Oleinik. Homogenization of Differential Operators and Integral Functionals. Springer Berlin Heidelberg, 1994. http://dx.doi.org/10.1007/978-3-642-84659-5.

[9] M. Karahan and Ö. Kalenderli. Coupled electrical and thermal analysis of power cables using finite element method. In Prof. Vyacheslav S. Vikhrenko(Ed.), Heat Transfer - Engineering Applications. InTech, 2011. http://dx.doi.org/10.5772/27350.

[10] U. Küttler and W.A. Wall. Fixed-point fluid-structure interaction solvers with dynamic relaxation. Computational Mechanics, 43(1):61-72, 2008. http://dx.doi.org/10.1007/s00466-008-0255-5.

[11] Y.Y. Li and J.Z. Cui. Two-scale analysis method for predicting heat transfer performance of composite materials with random grain distribution. Science in China Series A: Mathematics, 47(1):101-110, 2004. http://dx.doi.org/10.1360/04za0009.

[12] L. Liu, M. Huang, K. Yuan and M. Křižek. Numerical approximation of a nonlinear 3D heat radiation problem. Advances in Applied Mathematics and Mechanics, 1(1):125-139, 2009. 
[13] I. Makhkamova. Numerical Investigations of the Thermal State of Overhead Lines and Underground Cables in Distribution Networks. PhD thesis, School of Engineering and Computing Sciences of Durham University, 2010.

[14] R.L. Muddle, M. Mihajlović and M. Heil. An efficient preconditioner for monolithically-coupled large-displacement fluid-structure interaction problems with pseudo-solid mesh updates. Journal of Computational Physics, 231(21):7315-7334, 2012. http://dx.doi.org/10.1016/j.jcp.2012.07.001.

[15] J.H. Neher and M.H. McGrath. The calculation of the temperature rise and load capability of cable systems. Power Apparatus and Systems, Part III. Transactions of the American Institute of Electrical Engineers, 76(3):752-772, 1957. http://dx.doi.org/10.1109/AIEEPAS.1957.4499653.

[16] Y. Notay. An aggregation-based algebraic multigrid method. Report GANMN 08-02, 2009.

[17] OpenFOAM. Available from Internet: http://www.openfoam.org.

[18] I. Özdemir, W.A.M. Brekelmans and M.G.D. Geers. Computational homogenization for heat conduction in heterogeneous solids. International Journal for Numerical Methods in Engineering, 73(2):185-204, 2008. http://dx.doi.org/10.1002/nme.2068.

[19] J.R. Shewchuk. Delaunay refinement algorithms for triangular mesh generation. Computational Geometry: Theory and Applications, 22(1-3):21-74, 2002. http://dx.doi.org/10.1016/S0925-7721(01)00047-5. 16th $\{$ ACM $\}$ Symposium on Computational Geometry

[20] K. Stüben. A review of algebraic multigrid. Journal of Computational and Applied Mathematics, 128(1-2):281-309, 2001. http://dx.doi.org/10.1016/S0377-0427(00)00516-1. 